\title{
Front Matter: Volume 11187
}

, "Front Matter: Volume 11187," Proc. SPIE 11187, Optoelectronic Imaging and Multimedia Technology VI, 1118701 (26 December 2019); doi:

10.1117/12.2563101

SPIE. Event: SPIE/COS Photonics Asia, 2019, Hangzhou, China 


\title{
PROCEEDINGS OF SPIE
}

\section{Optoelectronic Imaging and Multimedia Technology VI}

\author{
Qionghai Dai \\ Tsutomu Shimura \\ Zhenrong Zheng \\ Editors
}

\section{1-23 October 2019 Hangzhou, China}

\author{
Sponsored by \\ SPIE \\ COS-Chinese Optical Society
}

\section{Cooperating Organizations}

Tsinghua University (China) • Peking University (China) • University of Science and Technology of China (China) • Zhejiang University (China) • Tianjin University (China) • Beijing Institute of Technology (China) • Beijing University of Posts and Telecommunications (China) • Nankai University (China) • Changchun University of Science and Technology (China) University of Shanghai for Science and Technology (China) • Capital Normal University (China) • Huazhong University of Science and Technology (China) • Beijing Jiaotong University (China) • China Jiliang University (China) • Shanghai Institute of Optics and Fine Mechanics, CAS (China) - Changchun Institute of Optics, Fine Mechanics and Physics, CAS (China) Institute of Semiconductors, CAS (China) • Institute of Optics and Electronics, CAS (China) • Institute of Physics, CAS (China) Shanghai Institute of Technical Physics, CAS (China) • China Instrument and Control Society (China) • Japan Optical Society (Japan) • Korea Optical Society (Korea, Republic of) • Australia Optical Society (Australia) - Singapore Optical Society (Singapore) • European Optical Society

\section{Supporting Organizations}

China Association for Science and Technology (CAST) (China)

Department of Information of National Nature Science Foundation, China (NSFC) (China)

Published by

SPIE

\section{Volume 11187}


The papers in this volume were part of the technical conference cited on the cover and title page. Papers were selected and subject to review by the editors and conference program committee. Some conference presentations may not be available for publication. Additional papers and presentation recordings may be available online in the SPIE Digital Library at SPIEDigitalLibrary.org.

The papers reflect the work and thoughts of the authors and are published herein as submitted. The publisher is not responsible for the validity of the information or for any outcomes resulting from reliance thereon.

Please use the following format to cite material from these proceedings:

Author(s), "Title of Paper," in Optoelectronic Imaging and Multimedia Technology VI, edited by Qionghai Dai, Tsutomu Shimura, Zhenrong Zheng, Proceedings of SPIE Vol. 11187 (SPIE, Bellingham, WA, 2019) Seven-digit Article CID Number.

ISSN: 0277-786X

ISSN: 1996-756X (electronic)

ISBN: 9781510630918

ISBN: 9781510630925 (electronic)

Published by

SPIE

P.O. Box 10, Bellingham, Washington 98227-0010 USA

Telephone +1 3606763290 (Pacific Time) · Fax +1 3606471445

SPIE.org

Copyright (C) 2019, Society of Photo-Optical Instrumentation Engineers.

Copying of material in this book for internal or personal use, or for the internal or personal use of specific clients, beyond the fair use provisions granted by the U.S. Copyright Law is authorized by SPIE subject to payment of copying fees. The Transactional Reporting Service base fee for this volume is $\$ 21.00$ per article (or portion thereof), which should be paid directly to the Copyright Clearance Center (CCC), 222 Rosewood Drive, Danvers, MA 01923. Payment may also be made electronically through CCC Online at copyright.com. Other copying for republication, resale, advertising or promotion, or any form of systematic or multiple reproduction of any material in this book is prohibited except with permission in writing from the publisher. The CCC fee code is $0277-$ $786 \mathrm{X} / 19 / \$ 21.00$.

Printed in the United States of America by Curran Associates, Inc., under license from SPIE.

Publication of record for individual papers is online in the SPIE Digital Library.

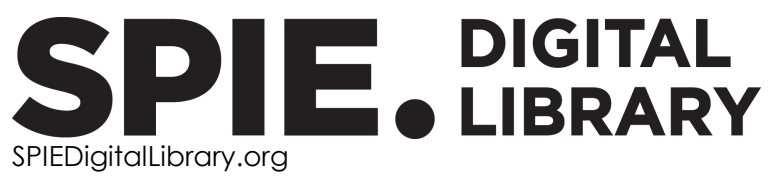

Paper Numbering: Proceedings of SPIE follow an e-First publication model. A unique citation identifier (CID) number is assigned to each article at the time of publication. Utilization of CIDs allows articles to be fully citable as soon as they are published online, and connects the same identifier to all online and print versions of the publication. SPIE uses a seven-digit CID article numbering system structured as follows:

- The first five digits correspond to the SPIE volume number.

- The last two digits indicate publication order within the volume using a Base 36 numbering system employing both numerals and letters. These two-number sets start with $00,01,02,03,04$, 05, 06, 07, 08, 09, OA, OB ... 0Z, followed by 10-1Z, 20-2Z, etc. The CID Number appears on each page of the manuscript. 


\title{
Contents
}

\author{
vii Authors \\ ix Symposium Committees \\ xiii Conference Committee
}

DEPTH AND LIGHT FIELD

$1118702 \quad$ High-resolution and real-time spectral-depth imaging with a compact system (Invited Paper) [11187-1]

1118703 Realizing high angular resolution multi-view and light-field displays with multi-projection technique [1 $11187-2]$

$1118704 \quad$ Monocular depth estimation based on unsupervised learning [11187-3]

1118705 A learning-based method using epipolar geometry for light field depth estimation [1 $1187-4]$

1118706 Light field SLAM based on ray-space projection model [1 $1187-5]$

\section{COMPUTER VISION}

$1118707 \quad$ Dynamic-stride-net: deep convolutional neural network with dynamic stride (Invited Paper) [1 $1187-6]$

$1118708 \quad$ No-reference image quality assessment based on an objective quality database and deep neural networks [1 $11187-7]$

$1118709 \quad$ Video quality assessment based on LOG filtering of videos and spatiotemporal slice images [11 $1187-8]$

11187 OA No-reference video quality assessment based on spatiotemporal slice images and deep convolutional neural networks [1 $11187-9]$

11187 OB An efficient stereo matching based on superpixel segmentation [11187-10]

\section{COMPUTATIONAL OPTICS}

11187 OD Optical coding of SPAD array and its application in compressive depth and transient imaging [11 187-13] 
$11187 \mathrm{OE} \quad$ Lensless wide-field imaging and depth sensing through a turbid scattering layer by round-trip field estimation [1 $11187-14]$

11187 OG Single-pixel depth imaging [11187-16]

\section{COMPUTATIONAL ACQUISITION AND ANALYSIS I}

11187 ol Joint-designed achromatic diffractive optics for full-spectrum computational imaging (Invited Paper) [1 $1187-17]$

11187 OK Hole filling algorithm for image array of one-dimensional integrated imaging [11187-19]

\section{COMPUTATIONAL ACQUISITION AND ANALYSIS II}

11187 OM High-SNR single-pixel phase imaging in the UV+VIS+NIR range (Invited Paper) [11187-21]

11187 ON Underwater image color correction algorithm based on scattering statistical characteristics [11 $1187-22]$

1118700 Effective 3D object reconstruction from densely sampled circular light fields [11187-23]

$111870 Q \quad$ No-reference quality assessment for synthesized images based on local geometric distortions [11187-25]

11187 OS Light field planar homography and its application [11187-27]

11187 OT Solving computer vision tasks with diffractive neural networks [1 $1187-70]$

\section{COMPUTER VISION I}

11187 OU Abnormal events detection method for surveillance video using an improved autoencoder with multi-modal input (Invited Paper) [1 $11187-28]$

11187 OV Multispectral demosaicing via non-local low-rank regularization [1 11 187-29]

11187 OW Attention-guided GANs for human pose transfer [11187-30]

11187 OY Supervoxel based point cloud segmentation algorithm [11187-32]

$111870 Z$ Intermediate deep-feature compression for multitasking [1 $1187-33]$

iv 
COMPUTER VISION II

1118710 Interactive gigapixel video streaming via multiscale acceleration (Invited Paper) [1 $1187-34]$

1118712 Semantic image inpainting with dense and dilated deep convolutional autoencoder adversarial network [1 $1187-36]$

1118713 Multiple hidden-targets recognizing and tracking based on speckle correlation method [1 $1187-37]$

IMAGE PROCESSING

1118714 Deep-learning for super-resolution full-waveform lidar (Invited Paper) [1 $1187-38]$

1118716 Viewport-adaptive 360-degree video coding using non-uniform tile for virtual reality communication [1 $1187-40]$

1118717 Cloud and snow detection from remote sensing imagery based on convolutional neural network [1 $1187-41]$

1118718 Surface defect recognition of varistor based on deep convolutional neural networks [11187-43]

\section{POSTER SESSION}

1118719 Facial action units recognition by de-expression residue learning [1 $1187-42]$

11187 1A Effect of defocus blur on the signal distribution of camera-based remote photoplethysmography [1 $11187-44]$

11187 1B Optical hash function based on the interaction between multiple scattering media and coherent radiation [1 $11187-45]$

11187 1C Binocular camera trap for wildlife detection [11187-46]

$11187 \mathrm{lE} \quad$ Infrared object image instance segmentation based on improved mask-RCNN [11187-48]

$11187 \mathrm{IF}$ Structural light 3D reconstruction algorithm based on deep learning [1 11 187-49]

$111871 G$ Spectral sensitivity estimation of color digital camera based on color checker [11187-50]

$11187 \mathrm{lH}$ An L0 regularized framelet based model for high-density mixed-impulse noise and Gaussian noise removal [1 $1187-51]$ 
$111871 \mathrm{~J} \quad$ Stereo matching using convolution neural network and LIDAR support point grid [11187-53]

$11187 \mathrm{lL} \quad$ Measuring the point spread function of a wide-field fluorescence microscope [1 $1187-55]$

$111871 \mathrm{~N} \quad$ A competition-based image saliency model [11187-57]

1118710 Generation of elemental image array based on photon mapping [11187-58]

$111871 P \quad$ Brain MRI image classification based on transfer learning and support vector machine [1 $1187-59]$

$111871 Q \quad$ Multifunctional image processor based on rank differences signals weighing-selection processing method and their simulation [1 $1187-60]$

11187 IR Fast bundle adjustment using adaptive moment estimation [11187-61]

$111871 \mathrm{~T} \quad$ A Lite Asymmetric DenseNet for effective object detection based on convolutional neural networks (CNN) [1 $11187-63]$

$111871 \mathrm{U} \quad$ Image inpainting using layered fusion and exemplar-based [11 187-65]

11187 IV BNU-LCSAD: a video database for classroom student action recognition [1 $1187-66]$

$111871 \mathrm{~W} \quad$ Improvement of semi-supervised learning in real application scenarios [11187-67]

$111871 \times \quad$ Efficient spectral confocal meta-lens in the near infrared [11187-69] 


\title{
Authors
}

Numbers in the index correspond to the last two digits of the seven-digit citation identifier (CID) article numbering system used in Proceedings of SPIE. The first five digits reflect the volume number. Base 36 numbering is employed for the last two digits and indicates the order of articles within the volume. Numbers start with 00, 01, 02, 03, 04, 05, 06, 07, 08, 09, OA, OB...0Z, followed by 10-1Z, 20-2Z, etc.

\author{
An, Ping, $0 Z, 1 U$ \\ Antonov, Alexander, $1 \mathrm{~J}$ \\ Belozubov, Alexander, $1 \mathrm{~J}$ \\ Bian, Liheng, OG, OM, OV, IR \\ Bo, Dezhi, 10 \\ Bo, Lei, 1E \\ Bykovskii, Sergei, $1 \mathrm{~J}$ \\ Cao, Xianbin, $1 \mathrm{R}$ \\ Chen, Fen, $\mathrm{ON}, \mathrm{OQ}$ \\ Chen, Hao, 10 \\ Chen, Hua, OY \\ Chen, Huasong, $1 \mathrm{H}$ \\ Chen, Ken, OY, 16 \\ Chen, Yixing, $1 \mathrm{X}$ \\ Dai, Qionghai, OT, OW, $1 \mathrm{~L}$ \\ Dai, Wenrui, 07 \\ Deng, Hai, $1 \mathrm{~T}$ \\ Denisov, Aleksei, 1J \\ Ding, Qin, $1 \mathrm{H}$ \\ Dong, Han, $1 \mathrm{C}$ \\ Du, Hongcai, 17 \\ Dun, Xiong, OD, Ol \\ Fan, Chunqi, 12, $1 \mathrm{~T}$ \\ Fan, Jingtao, OT, $1 \mathrm{~L}$ \\ Fan, Yuanyuan, $1 \mathrm{H}$ \\ Fang, LU, OT \\ Gong, Bo, 18 \\ Guo, Chengfei, OE \\ Guo, Jianhua, 17 \\ Guo, Peiyao, 10 \\ $\mathrm{Han}, \mathrm{Ke}, \mathrm{OB}$ \\ Han, Yixue, OK \\ He, Jun, 19, 1V, IW \\ $\mathrm{He}$, Sailing, IA \\ He, Shunfu, OE \\ He, Wenqi, 1B \\ Heidrich, Wolfgang, OD \\ $\mathrm{Hu}$, Limin, $1 \mathrm{~F}$ \\ Huang, Lin, 18 \\ Huang, Long, 12, $1 \mathrm{~T}$ \\ Huang, Xinpeng, $\mathrm{OZ}$ \\ Ji, Ting, OE \\ Jiang, Gangyi, OY, 16 \\ Jiang, $\mathrm{HaO}, 16$ \\ $\mathrm{Ke}$, Jun, 14 \\ Kormilitsyna, Elizaveta, $1 \mathrm{~J}$ \\ Krasilenko, Vladimir G., 1Q \\ Lan, Fangming, ON \\ Lazarev, Alexander A., 1Q
}

\author{
Lei, Pingshun, $1 \mathrm{C}$ \\ $\mathrm{Li}$, Haichao, OB \\ Li, Kun, OW, 17 \\ Li, Meng, OM \\ Li, Wei, OE \\ Li, Yang, $1 \mathrm{~N}$ \\ Li, Yaning, 06 \\ Li, Zimeng, $1 \mathrm{P}$ \\ Liang, Hongyao, $1 \mathrm{~F}$ \\ Liao, Meihua, 1B \\ Lin, Xing, OT \\ Liu, Gangping, 14 \\ Liu, Jietao, OE \\ Liu, Tiexin, $1 \mathrm{R}$ \\ Liu, Wan, 04, 05, $1 \mathrm{X}$ \\ Liu, Yebin, OW \\ Lu, Dajiang, IB \\ Ma, Ran, $1 \mathrm{U}$ \\ Ma, Xiaoyan, $0 Q$ \\ Ma, Yubing, $1 \mathrm{~L}$ \\ Ma, Zhan, 10 \\ Meng, Lisha, 12 \\ Meng, Yu, 10 \\ Mou, Xuanqin, 08, 09, 0A, 1N \\ Nan, Jing, $1 \mathrm{E}$ \\ Nikitovich, Diana V., $1 Q$ \\ Ning, Lei, $1 F$ \\ Ning, Siyu, $1 G$ \\ Niu, Haibin, $1 F$ \\ Peng, Xiang, 1B \\ Peng, Yifan, OD, Ol \\ Peng, Zongju, $\mathrm{ON}, \mathrm{OQ}$ \\ Qian, Qiaojian, 10 \\ Qiang, $\mathrm{HaO}, 1 \mathrm{H}$ \\ Qin, Zhentao, 05 \\ Ren, Kun, 12, 1T \\ Shao, Xiaopeng, OE, 13 \\ Shi, Junsheng, 1G \\ Shi, Qichao, ON \\ Shi, Yan, $1 F$ \\ Shimizu, Koichi, 13 \\ Song, Chenxi, OK \\ Song, Tengbiao, $1 \mathrm{~F}$ \\ Song, Zhengxi, 00 \\ Sun, Bo, 19, 1V, 1W \\ Sun, Liang, 1C \\ Sun, Qilin, OD \\ Sun, Xueying, 13 \\ Sun, Yan, 04, 05, $1 \mathrm{X}$
}


Surman, Phil, 03

Tao, Xiao, $1 \mathrm{X}$

Tian, Fujing, OY

Wang, Chang, $1 \mathrm{X}$

Wang, Gang, 13

Wang, Huayi, OG

Wang, Keke, $1 \mathrm{U}$

Wang, Qing, 06, 00, 0S

Wang, Shigang, OK, OU, 10, $1 \mathrm{P}$

Wang, Weiqian, $\mathrm{OZ}$

Wang, Xinwei, $1 \mathrm{C}$

Wang, Xucheng, 04, 05, $1 \mathrm{X}$

Wang, Xue, 06, OS

Wang, Yugang, OV

Wang, Zhanshan, 0 I

Wei, Jian, OK, OU

WU, Jiamin, OT

Wu, Qi, 00

Wu, Rengmao, $1 \mathrm{X}$

Wu, Yong, $1 \mathrm{~V}$

Wu, Yuxiang, $0 \mathrm{E}, 13$

Xia, Xinxing, 03

Xiao, Lei, 18

Xiao, Yongkang, 19, 1V, 1W

$\mathrm{Xie}, \mathrm{HaO}$, OT

Xie, Shaowei, 10

Xiong, Hongkai, 07

Xiong, Zhiwei, 02

$X U$, Feng, OT

$X u$, Yuhui, 07

$\mathrm{XU}$, Zhengnan, 10

$\mathrm{Xu}$, Zhongke, $1 \mathrm{C}$

Yan, Huanqing, IV, IW

Yan, Peng, 08, 09, 0A

Yan, Tao, OT

Yang, Chao, $\mathrm{OZ}$

Yang, Fuhao, ON

Yang, Jingyu, OW, 17

Yang, Libing, 00

Yang, Lin, 04, 05, 1X

Yang, Shuang, 1A

Yang, Tiejun, 18

Yang, Yang, $1 \mathrm{~F}$

Yang, Zerui, 07

Yao, Mingde, 02

YU, Lejun, 1V, IW

Yu, Mei, OY, 16

Yu, Xiaocui, 19

Yuan, Hanyi, $1 G$

Yue, Haichun, OU

Zhang, Aijia, 10

Zhang, Chenggong, 1B

Zhang, Jinsong, OW, 17

Zhang, Jun, OG, OM, OV, IR

Zhang, Lianbin, 1B

Zhang, Qi, 06, OS

Zhang, Xiangyu, 03

Zhang, Xiazhao, 08

Zhang, Yasong, $1 \mathrm{H}$
Zhao, Kaijie, IV, IW

Zhao, Yan, OU, 10, IP

Zhao, Yuyang, OW

Zhdanov, Andrey, $1 \mathrm{~J}$

Zhdanov, Dmitry, $1 \mathrm{~J}$

Zheng, Yuanjin, 03

Zheng, Zhenrong, 04, 05, $1 \mathrm{X}$

Zhou, Tiankuang, OT

Zhou, Yan, 1C

Zhou, Yufeng, 16

Zhu, Hao, $0 \mathrm{O}$

Zhu, Jinjin, OE

Zhu, Yudong, $1 \mathrm{~A}$

Zou, Wenhui, $0 Q$

viii

Proc. of SPIE Vol. 11187 1118701-8

Downloaded From: https://www.spiedigitallibrary.org/conference-proceedings-of-spie on 26 Apr 2023 Terms of Use: https://www.spiedigitallibrary.org/terms-of-use 


\title{
Symposium Committees
}

\author{
General Chairs
}

Jim M. Oschmann, President, SPIE and Ball Aerospace (United States)

Qihuang Gong, President, Chinese Optical Society and Peking

University (China)

General Co-chairs

Guangcan Guo, Past President, Chinese Optical Society and University of Science and Technology of China (China)

Zejin Liu, Vice President, Chinese Optical Society and National University of Defense Technology (China)

Technical Program Chairs

Ruxin Li, Vice President, Chinese Optical Society and Shanghai Institute of Optics and Fine Mechanics (China)

Xingde Li, Johns Hopkins University (United States)

Technical Program Co-chairs

Tianchu Li, National Institute of Metrology (China)

Wei Huang, Northwestern Polytechnical University (China)

Ying Gu, Vice President, Chinese Optical Society and PLA General Hospital (China)

Huilin Jiang, Changchun University of Science and Technology (China)

Wenqing Liu, Vice President, Chinese Optical Society, and Anhui Institute of Optics and Fine Mechanics (China)

Guobin Fan, China Academy of Engineering Physics (China)

Suotang Jia, Vice President, Chinese Optical Society, and Shanxi University (China)

Xiaomin Ren, Vice President, Chinese Optical Society, and Beijing University of Posts and Telecommunications (China)

Secretaries-General

Bo Gu, Deputy Secretary General, Chinese Optical Society (China)

Hong Yang, Deputy Secretary General, Chinese Optical Society and Peking University (China) 
Yan Li, Deputy Secretary General, Chinese Optical Society, and Peking University (China)

Daoxin Dai, Zhejiang University (China)

Local Organizing Committee Chair

Xu Liu, Secretary General, Chinese Optical Society and Zhejiang University (China)

Local Organizing Committee Co-chairs

Jianrong Qiu, Zhejiang University (China)

Daoxin Dai, Zhejiang University (China)

Local Secretaries

Wei Xiong, Chinese Optical Society (China)

Qing Yang, Zhejiang University (China)

Local Organizing Committee

Qing Yang, Zhejiang University (China)

Lan Wu, Zhejiang University (China)

Yaocheng Shi, Zhejiang University (China)

Dong Liu, Zhejiang University (China)

Yungui Ma, Zhejiang University (China)

Ke Si, Zhejiang University (China)

Yang Yang, Zhejiang University (China)

Xinyong Dong, China Jiliang University (China)

Le Wang, China Jiliang University (China)

Fei Tong, Chinese Optical Society (China)

Technical Organizing Committee

Mohammad Hossein Asghari, Loyola Marymount University

(United States) and Tachyonics Inc. (United States)

Pablo Benítez, Universidad Politécnica de Madrid (Spain)

Liangcai Cao, Tsinghua University (China)

P. Scott Carney, University of Rochester (United States)

Benyong Chen, Zhejiang University of Science and Technology

(China)

Hongqiang Chen, GE Global Research (United States)

Daoxin Dai, Zhejiang University (China)

Qionghai Dai, Tsinghua University (China)

Qihuang Gong, Peking University (China)

Ying Gu, Chinese PLA General Hospital (China) 
Guang-Can Guo, University of Science and Technology of China (China)

Byoung Seung Ham, Gwangju Institute of Science and Technology (Korea, Republic of)

Sen Han, University of Shanghai for Science and Technology (China) and Suzhou H\&L Instruments, LLC (China)

Zuyuan He, Shanghai Jiao Tong University (China)

Werner H. Hofmann, Technische Universität Berlin (Germany)

Minghui Hong, National University of Singapore (Singapore)

Bahram Jalali, University of California, Los Angeles (United States)

Satoshi Kawata, Osaka University (Japan)

Baojun Li, Jinan University (China)

Ming Li, Institute of Semiconductors, CAS (China)

Ruxin Li, Shanghai Institute of Optics and Fine Mechanics (China)

Xingde Li, Johns Hopkins University (United States)

Jian Liu, PolarOnyx, Inc. (United States)

Tiegen Liu, Tianjin University (China)

Yongfeng Lu, University of Nebraska-Lincoln (United States)

Qingming Luo, Huazhong University of Science and Technology

(China)

Gang-Ding Peng, The University of New South Wales (Australia)

Osamu Matoba, Kobe University (Japan)

Min Qiu, Westlake University (China)

Yuji Sano, ImPACT (Japan)

Yunlong Sheng, Université Laval (Canada)

Kebin Shi, Peking University (China)

Tsutomu Shimura, The University of Tokyo (Japan)

Upendra N. Singh, NASA Langley Research Center (United States)

Michael G. Somekh, Shenzhen University (China)

Yuguo Tang, Suzhou Institute of Biomedical Engineering and

Technology (China)

Masahiko Tani, University of Fukui (Japan)

Limin Tong, Zhejiang University (China)

Kazumi Wada, Massachusetts Institute of Technology (United States)

Yongtian Wang, Beijing Institute of Technology (China)

Rongshi Xiao, Beijing University of Technology (China)

Hongxing $X u$, Wuhan University (China)

Jianhua Yao, Zhejiang University of Technology (China)

Toru Yoshizawa, Tokyo University of Agriculture and Technology (Japan) and 3D Associates, Inc. (Japan)

Changyuan Yu, The Hong Kong Polytechnic University

(Hong Kong, China)

Xiao-Cong Yuan, Shenzhen University (China)

Cunlin Zhang, Capital Normal University (China)

Song Zhang, Purdue University (United States)

Xi-Cheng Zhang, University of Rochester (United States) 
Xinliang Zhang, Wuhan National Laboratory for Optoelectronics (China)

Xuping Zhang, Nanjing University (China)

Zhenrong Zheng, Zhejiang University (China)

Changhe Zhou, Shanghai Institute of Optics and Fine Mechanics (China)

Zhiping Zhou, Peking University (China)

Dan Zhu, Huazhong University of Science and Technology (China)

Ning Hua Zhu, Institute of Semiconductors, CAS (China) 


\title{
Conference Committee
}

\author{
Conference Chairs
}

Qionghai Dai, Tsinghua University (China)

Tsutomu Shimura, The University of Tokyo (Japan)

Zhenrong Zheng, Zhejiang University (China)

\section{Conference Program Committee}

Moshe Ben-Ezra, MIT Media Laboratory (United States)

Xudong Chen, National University of Singapore (Singapore)

Ya Cheng, Shanghai Institute of Optics and Fine Mechanics (China)

Jingtao Fan, Tsinghua University (China)

Jinwei Gu, Huawei Technologies Co., Ltd. (United States)

Yo-Sung Ho, Gwangju Institute of Science and Technology

(Korea, Republic of)

Bormin Huang, University of Wisconsin-Madison (United States)

Ivo Ihrke, INRIA Bordeaux (France)

Yoshiaki Kanamori, Tohoku University (Japan)

C. C. Jay Kuo, The University of Southern California (United States)

Kyros Kutulakos, University of Toronto (Canada)

Wanqing Li, University of Wollongong (Australia)

Xing Lin, University of California, Los Angeles (United States)

Yuan Luo, National Taiwan University (Taiwan, China)

Yifan Peng, Stanford University (United States)

Imari Sato, National Institute of Informatics (Japan) and Tokyo Institute of Technology (Japan)

Yoichi Sato, The University of Tokyo (Japan)

Yoav Yosef Schechner, Technion-Israel Institute of Technology (Israel)

John T. Sheridan, University College Dublin (Ireland)

Guangming Shi, Xidian University (China)

Guohai Situ, Shanghai Institute of Optics and Fine Mechanics (China)

Jinli Suo, Tsinghua University (China)

Lei Tian, Boston University (United States)

Gordon Wetzstein, Stanford University (United States)

Feng Wu, University of Science and Technology of China (China)

Bo Yang, University of Shanghai for Science and Technology (China)

Jingyi Yu, University of Delaware (United States)

Xiaolin Zhang, Shanghai Institute of Microsystem and Information

Technology (China) 


\section{Session Chairs}

1 Depth and Light Field

Mengqi Ji, Tsinghua University (China)

2 Computer Vision

Mengqi Ji, Tsinghua University (China)

3 Computational Optics

Jingtao Fan, Tsinghua University (China)

4 Computational Acquisition and Analysis I

Liheng Bian, Beijing Institute of Technology (China)

5 Computational Acquisition and Analysis II Jiamin Wu, Tsinghua University (China)

6 Computer Vision I

Jinli Suo, Tsinghua University (China)

7 Computer Vision II

Jingtao Fan, Tsinghua University (China)

$8 \quad$ Image Processing

Hui Qiao, Tsinghua University (China) 